\title{
End Points of Traumatic Brain Injury Resuscitation
}

\author{
Hadley K. H. Wesson ${ }^{1} \cdot$ Rahul Anand ${ }^{2} \cdot$ Paula Ferrada $^{2}$
}

Published online: 25 June 2015

(C) Springer International Publishing AG 2015

\begin{abstract}
Severe traumatic brain injury (TBI) is an insult severe enough to cause an acute and persistent loss of consciousness with a significant risk of death or disability. Worldwide, it is a leading cause of death, especially for younger people less than 50 years of age. Over the last two decades, we have made significant progress in our understanding of the pathophysiology of TBI. This however has not resulted in substantial improvements in outcome, as the incidence of TBI continues to increase and mortality rates remain relatively unchanged. To better understand the long-term outcome and end points in TBI resuscitation, tools to predict a patient's prognosis becomes essential. In this review, we discuss the role of clinical assessment, as such the Glasgow Coma Scale and the Full Outline of UnResponsiveness Score, neuroimaging, including computed tomography (CT) and MRI, and current research innovations, such as the biomarkers, to predict TBI outcomes. Additionally, we describe the clinical implications of using prognostic models, such as the International Mission on
\end{abstract}

This article is part of the Topical Collection on Blunt Head Trauma

Paula Ferrada

pferrada@mcvh-vcu.edu

Hadley K. H. Wesson

hkwesson@mcvh-vcu.edu

Rahul Anand

ranand@mcvh-vcu.edu

1 Department of General Surgery, Virginia Commonwealth University Medical Center, Richmond, VA 23225, USA

2 Division of Acute Care Surgical Services, Department of General Surgery, Virginia Commonwealth University Medical Center, Richmond, VA 23298, USA
Prognosis and Analysis of Clinical Trials in TBI (IMPACT) database models and the Corticosteroid Randomization after Significant Head Injury (CRASH) trial data models to calculate TBI outcome.

Keywords Traumatic brain injury (TBI) - Prognosis . Outcome $\cdot$ IMPACT $\cdot$ CRASH

\section{Introduction}

Severe traumatic brain injury (TBI) is an insult severe enough to cause an acute and persistent loss of consciousness with a significant risk of death or disability. It is a leading cause of death worldwide, especially for people less than 50 years of age [1]. In the USA, approximately $210-330$ patients with TBI are admitted to a hospital per 100,000 population each year [2•]. Thirty-nine percent of these patients with severe TBI die from their injury, and $60 \%$ have major disabilities [2•]. Over the last two decades, significant progress has been made to understand the pathophysiology of TBI. Unfortunately, this has not resulted in substantial improvements in outcome; the incidence of TBI continues to increase, and mortality rates remain relatively unchanged [3]. Our current knowledge of TBI end points of resuscitation can help predict neurologic outcome and may inform targeted therapies to improve mortality and morbidity.

Understanding and attempting to predict a patient's prognosis during the acute TBI resuscitation phase is critical: Such a prognosis can strongly influence clinical decisions and resource planning. In this review, we discuss end points in TBI resuscitation in terms of clinical assessment tools, as such the Glasgow Coma Scale (GCS), and the role of neuroimaging, including computed tomography (CT) and MRI. Additionally, we describe current research innovations, such as the role of 
biomarkers, and the clinical implications of using prognostic models to predict the outcome of TBI.

\section{Clinical Assessment}

There are three basic recovery processes in severe TBI: (1) the emergence of conscious awareness, (2) recovery of higher neuropsychological processing, and (3) the return of functional capacity [4•]. States of TBI can be described from most to least severe as brain death, coma, vegetative state, minimally conscious state, and emergence from minimally conscious state. In contrast to a vegetative state, patients in a minimally conscious state show signs of awareness of self or their environment. These signs however are not performed consistently or reliably. Even after emerging from a minimally conscious state, patients recovering from TBI may have significant neurological impairments, such as motor deficits, myoclonus, dystonia, movement disorders, or aphasia.

The key outcome predictor of TBI has historically been, and arguably continues to be, the physician's clinical assessment of injury severity [5]. Since its introduction in 1974, the GCS has been widely adopted as a simple method to numerically express the clinically observed features of consciousness (Table 1). Earlier studies from the 1980s found admission GCS score, when combined with age and pupillary response, was more than $80 \%$ accurate in predicting patient outcome as good, defined as no to moderate disability, or poor, defined as severe disability or persistent vegetative state $[7,8]$. A systematic review of prognostic factors associated with TBI found strong evidence that the GCS score at admission and the GCS motor score were significant predictors of outcome [9]. A recent multicenter retrospective analysis of 1173 patients with severe TBI found that the GCS score at the time of discharge from the ICU was a statistically significant predictor of 1-year outcome. Patients with a GCS score less than 10 at the time of discharge had a poor chance of a favorable outcome [10•].

The GCS score however has several limitations: It does not directly assess brain stem response, and the value of verbal and eye scores is irrelevant in patients who are intubated, are aphasic, or have injuries limiting eye movement [4•]. The Full Outline of Unresponsiveness (FOUR) scale attempts to address the limitations of GCS by removing verbal assessment and incorporating brain stem responses and breathing patterns (Table 1). When calculated at the time of the injury in the acute setting, the FOUR scale showed greater reliability than the GCS in patients with TBI, as described by a prospective observational study that showed a greater inter-rater reliability for the FOUR scale than GCS (FOUR: $\kappa=40.76, p<0.01$; GCS: $\kappa=40.59, p<0.0)$ [11]. However, in terms of longterm outcomes, a prospective study found that the FOUR scale's ability to predict poor functional outcomes at 6 months was equivalent to the GCS [12].

\section{Neuroimaging}

CT scan remains the investigation modality of choice to identify the presence and extent of acute TBI. In the setting of severe TBI, CT findings can show prognostic indicators, such as a midline shift, encroachment of basal cisterns, cerebral infarction, subarachnoid hemorrhage, intraventricular hemorrhage, diffuse injury, and extra-axial hematomas [13]. The widespread use of $\mathrm{CT}$ has led neuroimaging to be a key diagnostic modality in severe TBI.

In 1991, the Marshall classification of CT scan findings was devised to link specific CT findings with predictions of mortality. Currently, this classification is the most frequently used prognostic model and the one used to compare against newer models. It incorporates the anatomical nature of the injury to determine the outcome after acute TBI (Table 2). Using this classification, the extent of midline shift is the most important predictor of unfavorable outcome $[13,15]$.

The Marshall classification has been compared to the newer Rotterdam score, which reweighs the variables in the Marshall score and adds additional variables, such as the presence of intraventricular blood and subarachnoid hemorrhage (Table 3). One study found the Rotterdam score had a higher capacity to predict outcome, with an area under the receiver operating characteristic curve (AUC) of 0.77, compared to the Marshall score with an AUC of 0.67 [14]. Other studies found the Marshall score to be at least equal to that of the Rotterdam score, with both scores achieving an AUC of 0.85 [18]. Further studies may be needed to determine which scoring system is more predictive of mortality.

While CT scan during the acute post-TBI phase can be highly predictive of outcome, $38 \%$ of patients with a normal CT scan have an unfavorable outcome [19]. This is particularly pertinent with regard to diffuse axonal injury (DAI). DAI typically occurs during high-speed impact trauma that generates rotational acceleration, causing axonal damage through the tearing or stretching of the axons. Initially, these patients exhibit minor lesions or even no lesion on $\mathrm{CT}$, which cannot account for the severity of the clinical picture. There are three stages of DAI: Stage I includes involvement of the white-gray matter junction, stage II involves the corpus callosum, and stage III indicates involvement of the brain stem. Because both corpus callosum lesions and brain stem lesions are difficult to assess with $\mathrm{CT}$ scan, MRI is superior to $\mathrm{CT}$ in diagnosing DAI.

In addition to standard MRI, white matter damage, which is a key feature of TBI, can be identified and quantified with an MRI sequence known as diffusion tensor imaging (DTI). MRI DTI can show damage to critical areas such as the brain stem and corpus callosum, which correlate with poor prognosis. A 
Table 1 Clinically observed features of consciousness using GCS and FOUR scores

\begin{tabular}{|c|c|c|c|c|}
\hline \multicolumn{4}{|l|}{ Glasgow Coma Score (GCS) } & Score \\
\hline \multirow[t]{4}{*}{ Best eye response } & \multicolumn{3}{|l|}{ No eye opening } & 1 \\
\hline & \multicolumn{3}{|c|}{ Eye opening to pain } & 2 \\
\hline & \multicolumn{3}{|c|}{ Eye opening to verbal command } & 3 \\
\hline & \multicolumn{3}{|c|}{ Eyes open spontaneously } & 4 \\
\hline \multirow[t]{6}{*}{ Best motor response } & \multicolumn{3}{|c|}{ No motor response } & 1 \\
\hline & \multicolumn{3}{|c|}{ Extension to pain } & 2 \\
\hline & \multicolumn{3}{|l|}{ Flexion to pain } & 3 \\
\hline & \multicolumn{3}{|c|}{ Withdrawal from pain } & 4 \\
\hline & \multicolumn{3}{|l|}{ Localizes pain } & 5 \\
\hline & \multicolumn{3}{|l|}{ Obeys commands } & 6 \\
\hline \multirow[t]{5}{*}{ Best verbal response } & \multicolumn{3}{|c|}{ No verbal response } & 1 \\
\hline & \multicolumn{3}{|c|}{ Incomprehensible sounds } & 2 \\
\hline & \multicolumn{3}{|c|}{ Inappropriate words } & 3 \\
\hline & \multicolumn{3}{|l|}{ Confused } & 4 \\
\hline & \multicolumn{3}{|l|}{ Orientated } & 5 \\
\hline \multicolumn{5}{|c|}{ Full Outline of UnResponsiveness (FOUR) score } \\
\hline \multirow[t]{5}{*}{ Best eye response } & \multicolumn{3}{|c|}{ Eyelids closed to pain } & 0 \\
\hline & \multicolumn{3}{|c|}{ Eyelids closed by open to pain } & 1 \\
\hline & \multicolumn{3}{|c|}{ Eyelids closed but open to loud voice } & 2 \\
\hline & \multicolumn{3}{|c|}{ Eyelids open but not tracking } & 3 \\
\hline & \multicolumn{3}{|c|}{ Eyelids open and tracking on command } & 4 \\
\hline Motor response & Generalized myc & status & & 0 \\
\hline & No response to $\mathrm{p}$ & & & 0 \\
\hline & Extension respor & & & 1 \\
\hline & Flexion response & & & 2 \\
\hline & Localizes pain & & & 3 \\
\hline & Makes sign (i.e., & s-up) & & 4 \\
\hline Reflexes & Pupil reflexes & Corneal reflexes & Cough & \\
\hline & Absent & Absent & Absent & 0 \\
\hline & Absent & Absent & Present & 1 \\
\hline & Present & Absent & N/A & 2 \\
\hline & Absent & Present & N/A & 2 \\
\hline & One pupil fixed & Present & Present & 3 \\
\hline & Present & Present & Present & 4 \\
\hline Intubation and breathing & Intubation & Breathing & & \\
\hline & Intubated & Breathes at ventil & & 0 \\
\hline & Not intubated & Apnea & & 0 \\
\hline & Intubated & Breathes above $v$ & rate & 1 \\
\hline & Not intubated & Irregular & & 2 \\
\hline & Not intubated & Cheyne-Stokes & & 3 \\
\hline & Not intubated & Regular & & 4 \\
\hline
\end{tabular}

Source: Wijdicks et al. [6] prospective observational multicenter cohort study of 105 patients with TBI showed that the ability to assess the extent and severity of white matter injury using MRI DTI is a major predictor of outcome after severe TBI [20]. Neuroimaging findings from CT, MRI, and the newer MRI DTI are powerful tools to predict the outcome and end points of TBI resuscitation.

\section{Monitoring the Injured Brain}

Continuous monitoring of patients with severe TBI provides information to prevent and treat cerebral ischemia. Monitoring intracranial pressure (ICP) and cerebral perfusion pressure (CPP) is standard practice. Sustained elevations of ICP greater 
Table 2 Marshall classification of CT scan findings

\begin{tabular}{|c|c|c|}
\hline \multicolumn{3}{|l|}{ Marshall classification } \\
\hline \multicolumn{2}{|l|}{ Findings } & Score \\
\hline \multicolumn{2}{|l|}{ No visible intracranial pathology } & Diffuse injury I \\
\hline \multicolumn{2}{|c|}{$\begin{array}{l}\text { Cisterns are present with midline shift of } 0-5 \mathrm{~mm} \text { and/or lesion densities } \\
\text { present; no high or mixed density lesion }>25 \mathrm{~cm}^{3} \text { may include bone frag- } \\
\text { ments and foreign bodies }\end{array}$} & Diffuse injury II \\
\hline \multicolumn{2}{|c|}{ Cisterns compressed or absent with midline shift of $0-5 \mathrm{~mm}$} & Diffuse injury III \\
\hline \multicolumn{2}{|l|}{ No high or mixed density lesion $>25 \mathrm{~cm}^{3}$} & Diffuse injury IV \\
\hline \multicolumn{2}{|l|}{ No high or mixed density lesion $>25 \mathrm{~cm}^{3}$} & Evacuated mass lesion \\
\hline \multicolumn{2}{|l|}{ High or mixed density lesion $>25 \mathrm{~cm}^{3}$} & Non-evacuated mass lesion \\
\hline \multicolumn{3}{|l|}{ Not surgically evacuated } \\
\hline Injury & Findings & Score \\
\hline \multirow[t]{3}{*}{ Basal cisterns } & Normal & 0 \\
\hline & Compressed & 1 \\
\hline & Absent & 2 \\
\hline \multirow[t]{3}{*}{ Midline shift } & Normal & 0 \\
\hline & Compressed & 1 \\
\hline & Absent & 2 \\
\hline \multirow[t]{2}{*}{ Epidural mass lesion } & No shift or $\leq 5 \mathrm{~mm}$ & 0 \\
\hline & Shift $>5 \mathrm{~mm}$ & 1 \\
\hline \multirow[t]{2}{*}{ Intraventricular blood or traumatic SAH } & Absent & 0 \\
\hline & Present & 1 \\
\hline
\end{tabular}

Source: Maas et al. [14]

SAH subarachnoid hemorrhage

${ }^{a}$ Interpretation of Rotterdam score: The final score is the sum of the scoring items, associated with mortality at 6 months post injury of score $1=0 \%$, score $2=7 \%$, score $3=16 \%$, score $4=26 \%$, score $5=53 \%$, score $6=61 \%$ than $20 \mathrm{mmHg}$ or CPP less than 50-60 mmHg are linked with cerebral infarction, herniation, and death following TBI [21]. However, even when ICP and CPP are within normal limits, significant reductions in brain tissue oxygen pressure may occur, leading to worse outcomes [22]. As such, studies support the use of clinical tools to monitor brain tissue oxygen pressure. These tools include brain tissue oximetry, transcranial Doppler (TCD) ultrasound, microdialysis, and continuous electroenchephalography (cEEG).

In addition to following ICP and CPP trends, brain tissue oxygenation $\left(\mathrm{BtipO}_{2}\right)$ may add information regarding end points of TBI resuscitation. By monitoring levels of $\mathrm{BtipO}_{2}$, it is hypothesized that CPP levels can be optimized on an individual basis [23]. After a TBI, the partial pressure of oxygen in brain tissue $\left(\mathrm{PbrO}_{2}\right)$ increases with elevations in CPP; this increase relative to an increase in arterial $\mathrm{PO}_{2}$ is termed brain tissue oxygenation. Brain oxygen tension can be continuously measured with an invasive sensor probe, such as Clark-type electrode. Normal $\mathrm{PbrO}_{2}$ ranges between 35 and $50 \mathrm{mmHg}$, suggesting that ischemic thresholds lie between 5 and $20 \mathrm{mmHg}$; the true cutoff level is still under investigation $[23,24]$. As more studies show that using brain tissue oximetry in addition to ICP and CPP monitoring leads to better outcomes, the evidence to include brain tissue oximetry as a means to determine end point resuscitation in TBI is increasing [24].

Studies have also shown that using TCD to monitoring cerebral blood flow is useful in measuring high pulsatility index, defined as the difference between systolic and diastolic blood flow velocities divided by the mean blood flow velocity. A high pulsatility index, in turn, is strongly associated with a worse outcome and higher mortality [25]. A prospective observational study of 24 patients with severe TBI found that the use of early TCD at the time of hospital admission allowed physicians to identify severely brain-injured patients with brain hypoperfusion and implement an early TCD goaldirected therapy to restore normal cerebral perfusion. The study postulates that this could lead to a decrease in the extent of secondary brain injury and may be used as an end point in TBI resuscitation [26].

Microdialysis uses concentrations of pyruvate, glucose, glycerol, and lactate in the extracellular fluid to analyze focal brain biochemistry. Using microdialysis, a cohort study of 223 patients with severe TBI found that low brain extracellular glucose and elevated lactate-to-pyruvate ratio were independent predictors of mortality at 6 months [27]. A more recent 
Table 3 IMPACT and CRASH models for the prediction of mortality and unfavorable outcome

\begin{tabular}{lll}
\hline International Mission on Prognosis and Analysis of Clinical Trials in TBI (IMPACT) & \\
Basic model & Core model & Lab model \\
Age & Age & Age \\
GCS, motor score & GCS, motor score & GCS \\
Pupillary reactivity & Pupillary reactivity & Pupillary reactivity \\
& CT: epidural hematoma & CT: epidural hematoma \\
& CT: traumatic SAH & CT: traumatic SAH \\
& Hypoxia & Hypoxia \\
& Hypotension & Hypotension \\
Corticoid Randomization After Significant & Head Injury (CRASH) & Blood glucose level \\
Basic model & Extended model & Hemoglobin level \\
Age & Age & \\
GCS, motor score & GCS, motor score & \\
Pupillary reactivity & Pupillary reactivity & \\
Motor extracranial injury & Motor extracranial injury & \\
& CT: traumatic SAH & \\
\hline
\end{tabular}

Source: IMPACT group [16]; MRC Crash Trial Collaborators [17]

$S A H$ subarachnoid hemorrhage but smaller study of 14 patients found that an increase in lactate glucose in CSF significantly correlates with an unfavorable outcome. Interestingly, the lactate-to-pyruvate ratio was not statistically significant [28]. Additional studies are needed to further validate these findings and the role of microdialysis.

Seizures can result in hippocampal atrophy worsening the trauma-induced injury [29]. The use of cEEG shows that seizure activity and status epilepticus occur in a significant number of patients with severe TBI and are linked to a poor outcome. In a study of 94 patients who underwent cEEG monitoring, 21 patients had seizures; $100 \%$ of patients with status epilepticus died [30]. Current guidelines recommend that patients with moderate to severe TBI be treated with 7 days of seizure prophylaxis [31]. Further research is needed to determine if early suppression of seizure activity may improve outcome after TBI.

\section{Prognostic Models}

Two TBI prognostic tools, the International Mission on Prognosis and Analysis of Clinical Trials in TBI (IMPACT) database models and the Corticosteroid Randomization after Significant Head Injury (CRASH) trial data models, were developed using multiple logistic regression models to predict functional outcomes after a TBI $[32,33]$. The IMPACT models were developed from a database of 11 studies comprised of 8509 patients with moderate or severe TBI. In IMPACT, three models - the basic model, the core model, and the lab model-were developed for the prediction of mortality and unfavorable outcome, defined as death, vegetative state, and severe disability at 6 months (Table 3 ) [32].

The CRASH models were developed from a database of 7526 patients with mild, moderate, or severe TBI. Two different models, the basic model and the extended model, were developed to predict mortality at 2 weeks after injury and unfavorable outcome at 6 months after injury (Table 3 ). With an increased complexity, the extended model performed slightly superior to the basic model. Regardless of the model used, the three key predictors of outcome were age, GCS motor score, and pupillary reactivity [34••]. Both IMPACT and CRASH models were tested for external validation against five independent data sets, which found no relevant difference in performance between the IMPACT and CRASH models [34••].

Using IMPACT data, the models' authors constructed a prognosis calculator in an attempt to predict the 6-month outcome in patients with moderate to severe TBI. The calculator uses variables from the lab IMPACT model [16]. A singlecenter study of 48 patients with severe TBI showed that the IMPACT prognosis calculator leads to an overestimation of mortality and unfavorable outcome with an absolute risk reduction for mortality of $13.6 \%$ compared to using the IMPACT database alone [35]. It is thus recommended that the IMPACT prognosis calculator be used cautiously when predicting moderate to severe TBI outcome $[35,36]$. 
The authors of the CRASH models also developed a calculator for the purposes of estimating an individual's outcome following severe TBI [17]. Similar to the IMPACT prognosis calculator, multiple studies found that the CRASH prognosis calculator overestimates the risk of unfavorable outcomes. These studies show that the calculator is only accurate for a risk level above $75 \%$ for an unfavorable outcome; otherwise, the accuracy of the calculator is questionable [37-40]. As such, some studies do not recommend using the CRASH calculator for individual treatment decisions.

The main differences between the IMPACT and CRASH models are the inclusion variables and the study population. The CRASH models include major extracranial injury as a predictor of outcome, while the IMPACT models do not. However, in an external review, the added prognostic effect of major extracranial injury was negligible, suggesting this difference to be relatively insignificant [34••]. In terms of the study population, the IMPACT models were developed using patients with moderate to severe TBI from high-income countries; the CRASH models included patients with mild to severe TBI in low- and middle-income countries (LMICs). As such, the CRASH models may be more preferable for patients with mild TBI or patients from LMICs.

\section{Research Innovations: Biomarkers}

Despite improvements in the management of patients with TBI and improved modalities to predict outcome, mortality and morbidity remain high. New prognostic tools, such as proteomic biomarkers, are currently being researched. The potential role of biomarkers is based on the concept that the extent of brain injury is determined by the severity of the primary mechanical injury and the consequences of secondary biomolecular injury. These biomolecular cascades, in turn, cause neuroinflammation, leading to cerebral edema, enhanced ICP, and delayed cellular damage [41].

Cytokines are critical mediators of neuroinflammation after TBI. Inflammatory cytokines stimulate astrocytes, which increase neuroinflammation and secondary injury after trauma [41]. A prospective single-center study of 93 patients with GCS $<9$ following TBI found interleukin-10 (IL-10) to be an independent predictor of severe TBI prognosis. Elevated serum IL-10 correlated significantly with GCS severity and with hospital mortality in patients with severe TBI [42].

Other studies show that serum levels of S100 beta protein and neuron-specific enolase, markers of glial and neuronal damage, respectively, correlate with TBI severity [43-45]. Thus, measuring the S100 beta protein could be useful in evaluating the severity of TBI and determining the long-term prognosis [46]. Biomarkers such as IL-10, S100, and enolase are still under investigation as questions remain regarding the optimal biochemical method, timing of the sample, and prognostic threshold.

\section{Conclusions}

In TBI, the purpose of accurate, accessible, and reliable prognostic tools is to inform treatment strategies and determine end points in resuscitation. In this review, we explored the role of clinical assessment, with particular reference to the role of the GSC and FOUR scores to predict poor outcome. We reviewed the role of CT scan and classification strategies that estimate poor outcomes. We also reviewed the role of MRI in settings such as DAI. We discussed the current limitations of continuous monitoring tools including ICP and CPP and proposed how newer technologies such as brain tissue oximetry, TCD ultrasound, microdialysis, and cEE may be useful in assessing brain tissue oxygen pressure as a marker of poor outcomes. We described the IMPACT and CRASH prognostic models and reviewed the evidence that shows that both the IMPACT and CRASH prognostic calculators should be used with caution, as they can overestimate a poor outcome in the individual patients. Finally, we described future areas of research, such as the role of IL-10 and S100 beta protein biomarkers. Yet, these existing methods to predict end points of resuscitation and recovery after severe TBI still lack accuracy. Further research will be key to developing new treatments and diagnostic tools to better understand the etiology and prognosis of TBI and ultimately lead to improved mortality and morbidity.

\section{Compliance with Ethics Guidelines}

Conflict of Interest Hadley K. H. Wesson, Rahul Anand, and Paula Ferrada declare no conflicts of interest.

Human and Animal Rights and Informed Consent This article does not contain any studies with human or animal subjects performed by any of the authors.

\section{References}

Papers of particular interest, published recently, have been highlighted as:

- Of importance

- Of major importance

1. Maas AI, Stocchetti N, Bullock R. Moderate and severe traumatic brain injury in adults. Lancet Neurol. 2008;7:728-41.

2. Rosenfeld JV, Maas AI, Bragge P, et al. Early management of severe traumatic brain injury. Lancet. 2012;380:1088-98. This review article explores the role of decompressive craniectomy in the setting of TBI, potential pharmicologic agents, such as erythropoietin, statins, and tranexamic acid, and invasive monitoring, such as cerebral oximetry and microdialysis.

3. Stein SC, Georgoff P, Meghan S, et al. 150 years of treating severe traumatic brain injury: a systematic review of progress in mortality. J Neurotrauma. 2010;27:1343-53. 
4. Stevens RD, Sutter R. Prognosis in severe brain injury. Crit Care Med. 2013;41:1104-23. This review article explored updates on neurologic prognosis following traumatic brain injury and hypoxic-ischemic encephalopathy following cardiac arrest.

5. Lingsma HF, Roozenbeek B, Steyerberg EW, et al. Early prognosis in traumatic brain injury: from prophecies to predictions. Lancet Neurol. 2010;9:543-54.

6. Wijdicks EFM, Bamler WR, et al. Validation of a new coma scale: the FOUR score. Ann Neurol. 2005;58:585-93.

7. Choi SC, Ward JD, Becker DP. Chart for outcome prediction in severe head injury. J Neurosurg. 1983;59:294-7.

8. Narayan RK, Greenberg RP, Miller JD, et al. Improved confidence of outcome prediction in severe head injury. A comparative analysis of the clinical examination, multimodality evoked potentials, Ct scanning, and intracranial pressure. J Neurosurg. 1981;54:751-62.

9. Husson EC, Ribbers GM, Willemse-van Son AH, et al. Prognosis of Six-month functioning after moderate to severe traumatic brain injury: a systematic review of prospective cohort studies. J Rehabil Med. 2010;42:425-36.

10. Leitgeb J, Mauritz W, Brazinova A, et al. Glasgow coma scale score at intensive care unit discharge predicts the 1-year outcome of patients with severe traumatic brain injury. Eur J Trauma Emerg Surg. 2013;39:285-92. This retropspective study finds the GCS score at the time of ICU discharge is a good predictor of TBI outcome at 1-year, with a GCS $<10$ consitent with an unfavorable outcome.

11. Kevric J, Jelinek GA, Knott J, et al. Validation of the full outline of unresponsiveness (four) scale for conscious state in the emergency department: comparison against the Glasgow coma scale. Emerg Med J. 2011;28:486-90.

12. Sadaka F, Patel D, Lakshmanan R. The four score predicts outcome in patients after traumatic brain injury. Neurocrit Care. 2012;16:95101.

13. Zhu GW, Wang F, Liu WG. Classification and prediction of outcome in traumatic brain injury based on computed tomographic imaging. J Int Med Res. 2009;37:983-95.

14. Maas AI, Hukkelhoven CW, Marshall LF, et al. Prediction of outcome in traumatic brain injury with computed tomographic characteristics: a comparison between the computed tomographic classification and combinations of computed tomographic predictors. Neurosurgery. 2005;57:1173-82. discussion 1173-1182.

15. Nelson DW, Nystrom H, MacCallum RM, et al. Extended analysis of early computed tomography scans of traumatic brain injured patients and relations to outcome. J Neurotrauma. 2010;27:51-64.

16. IMPACT group. Impact: International Mission for Prognosis and Analysis of Clinical Trials in Tbi. 2014. Available at: http://www. tbi-impact.org. Accessed 15 Oct 2014.

17. MRC Crash Trial Collaborators. Head Injury Prognosis. 2007. Available at: http://www.trialscoordinatingcentre.lshtm.ac.uk/ Riskcalculator/index.html. Accessed 15 Oct 2014.

18. Mata-Mbemba D, Mugikura S, Nakagawa A, et al. Early CT findings to predict early death in patients with traumatic brain injury: Marshall and Rotterdam CT scoring systems compared in the major academic tertiary care hospital in Northeastern Japan. Acad Radiol. 2014;21:605-11.

19. Calvi MR, Beretta L, Dell'Acqua A, et al. Early prognosis after severe traumatic brain injury with minor or absent computed tomography scan lesions. J Trauma. 2011;70:447-51.

20. Galanaud D, Perlbarg V, Gupta R, et al. Assessment of white matter injury and outcome in severe brain trauma: a prospective multicenter cohort. Anesthesiology. 2012;117:1300-10.

21. Marino R, Gasparotti R, Pinelli L, et al. Posttraumatic cerebral infarction in patients with moderate or severe head trauma. Neurology. 2006;67:1165-71.
22. Chang JJ, Youn TS, Benson D, et al. Physiologic and functional outcome correlates of brain tissue hypoxia in traumatic brain injury. Crit Care Med. 2009;37:283-90.

23. Purins K, Lewen A, Hillered L, et al. Brain tissue oxygenation and cerebral metabolic patterns in focal and diffuse traumatic brain injury. Front Neurol. 2014;5:64.

24. Prabhakar H, Sandhu K, Bhagat H, et al. Current concepts of optimal cerebral perfusion pressure in traumatic brain injury. J Anaesthesiol Clin Pharmacol. 2014;30:318-27.

25. Moreno JA, Mesalles E, Gener J, et al. Evaluating the outcome of severe head injury with transcranial doppler ultrasonography. Neurosurg Focus. 2000;8, e8.

26. Ract $\mathrm{C}$, Le Moigno S, Bruder N, et al. Transcranial doppler ultrasound goal-directed therapy for the early management of severe traumatic brain injury. Intensive Care Med. 2007;33:645-51.

27. Timofeev I, Carpenter KL, Nortje J, et al. Cerebral extracellular chemistry and outcome following traumatic brain injury: a microdialysis study of 223 patients. Brain. 2011;134:484-94.

28. Thelin EP, Nelson DW, Ghatan PH, et al. Microdialysis monitoring of CSF parameters in severe traumatic brain injury patients: a novel approach. Front Neurol. 2014;5:159.

29. Vespa PM, McArthur DL, Xu Y, et al. Nonconvulsive seizures after traumatic brain injury are associated with hippocampal atrophy. Neurology. 2010;75:792-8.

30. Vespa PM, Nuwer MR, Nenov V, et al. Increased incidence and impact of nonconvulsive and convulsive seizures after traumatic brain injury as detected by continuous electroencephalographic monitoring. J Neurosurg. 1999;91:750-60.

31. Brain Trauma F, American Association of Neurological S, Congress of Neurological S, et al. Guidelines for the management of severe traumatic brain injury. X. brain oxygen monitoring and thresholds. J Neurotrauma 2007;24 Suppl 1:S65-70.

32. MRC Crash Trial Collaborators, Perel P, Arango M, et al. Predicting outcome after traumatic brain injury: practical prognostic models based on large cohort of international patients. BMJ. 2008;336: 425-9.

33. Steyerberg EW, Mushkudiani N, Perel P, et al. Predicting outcome after traumatic brain injury: development and international validation of prognostic scores based on admission characteristics. PLoS Med. 2008;5, e165. discussion e165.

34.• Roozenbeek B, Lingsma HF, Lecky FE, et al. Prediction of outcome after moderate and severe traumatic brain injury: external validation of the international mission on prognosis and analysis of clinical trials (Impact) and Corticoid Randomisation after Significant Head Injury (Crash) Prognostic models. Crit Care Med. 2012;40:160917. This external validation study compared the IMPACT and the CRASH prognostic models to predict outcome after moderate or severe traumatic brain injury.

35. Olivecrona M, Koskinen LO. The impact prognosis calculator used in patients with severe traumatic brain injury treated with an Icptargeted therapy. Acta Neurochir (Wien). 2012;154:1567-73.

36. Honeybul S, Ho KM. Use of the crash study prognosis calculator in patients with severe traumatic brain injury. J Clin Neurosci. 2013;20:1808-10.

37. Honeybul S, Ho KM, Lind CR, et al. Observed versus predicted outcome for decompressive craniectomy: a population-based study. J Neurotrauma. 2010;27:1225-32.

38. Honeybul S, Ho KM, Lind $\mathrm{CR}$, et al. The retrospective application of a prediction model to patients who have had a decompressive craniectomy for trauma. J Neurotrauma. 2009;26:2179-83.

39. Honeybul S, Ho KM, Lind CR, et al. Decompressive craniectomy for neurotrauma: the limitations of applying an outcome prediction model. Acta Neurochir (Wien). 2010;152:959-64.

40. Olivecrona $\mathrm{M}$, Olivecrona $\mathrm{Z}$. Use of the crash study prognosis calculator in patients with severe traumatic brain injury treated with 
an intracranial pressure-targeted therapy. J Clin Neurosci. 2013;20: 996-1001.

41. Schwarzbold ML, Rial D, De Bem T, et al. Effects of traumatic brain injury of different severities on emotional, cognitive, and oxidative stress-related parameters in mice. J Neurotrauma. 2010;27: 1883-93.

42. Schneider Soares FM, Menezes de Souza N, Liborio Schwarzbold M, et al. Interleukin-10 is an independent biomarker of severe traumatic brain injury prognosis. Neuroimmunomodulation. 2012;19:377-85.

43. Vos PE, Jacobs B, Andriessen TM, et al. Gfap and S100b are biomarkers of traumatic brain injury: an observational cohort study. Neurology. 2010;75:1786-93.
44. Wiesmann M, Steinmeier E, Magerkurth O, et al. Outcome prediction in traumatic brain injury: comparison of neurological status, $\mathrm{Ct}$ findings, and blood levels of S100b and gfap. Acta Neurol Scand. 2010;121:178-85.

45. Bohmer AE, Oses JP, Schmidt AP, et al. Neuron-specific enolase, $\mathrm{S} 100 \mathrm{~b}$, and glial fibrillary acidic protein levels as outcome predictors in patients with severe traumatic brain injury. Neurosurgery. 2011;68:1624-30. discussion 1630-1621.

46. Mercier E, Boutin A, Lauzier F, et al. Predictive value of S-100beta protein for prognosis in patients with moderate and severe traumatic brain injury: systematic review and meta-analysis. BMJ. 2013;346: f1757. 